\title{
Ductus Arteriosus Shunts in the Respiratory Distress Syndrome
}

\author{
N.R.C.Roberton ${ }^{[30]}$ and G.W.Dahlenburg
}

The Nuffield Neonatal Research Unit, Institute of Child Health, Hammersmith Hospital, London, England

\begin{abstract}
Extract
The arterial blood gas tensions in the right radial artery and the lower abdominal aorta were compared in 21 infants suffering from respiratory distress syndrome. Simultaneous sampling was carried out using a radial artery catheter inserted by a cut-down technique and an umbilical arterial catheter inserted into the abdominal aorta. One hundred thirty-nine simultaneous blood samples were analysed for $\mathrm{paO}_{2}, \mathrm{pH}$, and $\mathrm{pCO}_{2}$. In no case was there any significant difference in $\mathrm{pH}$ and $\mathrm{pCO}_{2}$ values in samples of blood drawn from the two sites. Differences in $\mathrm{paO}_{2}$ were observed but owing to inherent errors in measurement, only differences of greater than $10 \mathrm{~mm} \mathrm{Hg}$ were regarded as significant. In 27 out of the 139 determinations, $\mathrm{paO}_{2}$ from the right radial artery exceeded that from the abdominal aorta by more than $10 \mathrm{~mm} \mathrm{Hg}$. The babies studied were divided into the following groups: Group 1, 6 babies with no significant $\mathrm{paO}_{2}$ difference between the two sampling sites. Group 2(a), 6 babies with significantly higher oxygen tensions in blood drawn from the radial artery but only at aortic $\mathrm{paO}_{2}$ levels of more than $140 \mathrm{~mm} \mathrm{Hg}$. In this group there were no significant differences when the abdominal aorta $\mathrm{paO}_{2}$ was less than $140 \mathrm{~mm} \mathrm{Hg}$. Group 2(b), 3 babies with slightly higher radial artery oxygen tensions only when they were being ventilated with a positive pressure respirator. Group 2(c), 6 babies with significantly higher $\mathrm{paO}_{2}$ levels in the radial artery at arterial oxygen tensions of less than $100 \mathrm{~mm} \mathrm{Hg}$. In two of these babies, the right radial $\mathrm{paO}_{2}$ (and therefore presumably the retinal artery $\mathrm{paO}_{2}$ ) would have exceeded $160 \mathrm{~mm} \mathrm{Hg}$ had the ambient oxygen concentration been raised sufficiently to produce an aorta $\mathrm{paO}_{2}$ of $100 \mathrm{~mm} \mathrm{Hg}$ and had the total shunt and the proportion passing through the ductus arteriosus remained constant. In two of these babies, however, increasing ambient oxygen concentration decreased the total shunt and the proportion of the shunt passing through the ductus. In the remaining four babies in Group 2(c), the severity of the disease was such that it was impossible to raise the aorta $\mathrm{paO}_{2}$ to $100 \mathrm{~mm} \mathrm{Hg}$.
\end{abstract}

\section{Speculation}

From these data it seems that most of the $\mathrm{R} \rightarrow \mathrm{L}$ shunt in respiratory distress syndrome must pass through the foramen ovale or the lungs. Only rarely does more than $15 \%$ of the $\mathrm{R} \rightarrow \mathrm{L}$ shunt pass through the ductus arteriosus.

The final quantitative differentiation between shunting in the lungs and through the foramen ovale is most likely to be made by dye dilution studies from peripheral vein or right atrial injections. 


\section{Introduction}

It has been established that the major cause of the hypoxemia in respiratory distress syndrome (RDS) is a net right to left shunt of cardiac output $[26,27]$. There are three possible sites for the shunt: the foramen ovale, the ductus arteriosus, and the lungs, either by direct vascular bypass through the bronchial circulation or by pulmonary arterial blood coming into contact with unventilated alveoli. There is good qualitative evidence that bidirectional shunts occur in all these sites [12, 17, 25]. During the first 24-48 hours of life, the pulmonary artery pressure is close to systemic levels and falls steadily during this time [5]; nevertheless, $\mathrm{R} \rightarrow \mathrm{L}$ shunting through the ductus has been demonstrated in normal newborn babies at this age $[4,16,19]$. In the hypoxic neonate with RDS, the pulmonary artery pressure is likely to rise and closure of the ductus will be delayed $[2,13,15,23]$; both these factors will increase the likelihood of a $\mathrm{R} \rightarrow \mathrm{L}$ shunt occurring through the ductus arteriosus. In very sick babies with RDS studied by cardiac catheterization, however, net $\mathrm{R} \rightarrow \mathrm{L}$ shunts through the ductus arteriosus were not demonstrated [22].

In recent years, measurement of arterial oxygen tension has been used as a guide to oxygen therapy in newborn infants with respiratory illnesses. The umbilical artery has provided the most convenient source of arterial blood. Umbilical arterial catheters are usually placed to lie in the abdominal aorta and will therefore be sampling post-ductal blood. One purpose of measuring $\mathrm{paO}_{2}$ is to prevent dangerously high levels that might cause retrolental fibroplasia. If there is a net $\mathrm{R} \rightarrow \mathrm{L}$ shunt through the ductus arteriosus, blood reaching the retina will have a higher oxygen tension than that in the abdominal aorta, and dangerously high retinal levels could occur at normal aortic $\mathrm{paO}_{2}$ values. Stahlman [25] and Oliver et al. [18] have reported babies who had much higher $\mathrm{paO}_{2}$ levels in left atrial blood than in peripheral arterial samples. Recently, Genu et al. [3] have reported the presence of $R \rightarrow L$ shunts at a ductus level in 14 babies by comparing temporal artery and abdominal aorta $\mathrm{pO}_{2}$ values.

Thus, when using abdominal aorta $\mathrm{paO}_{2}$ as a guide to ambient oxygen concentration, there is a risk of being misled, in the presence of substantial $\mathrm{R} \rightarrow \mathrm{L}$ shunts through the ductus arteriosus, and of subjecting babies to dangerously high retinal blood oxygen tensions.

In order to study this problem, we supplemented our routine management of babies with respiratory distress [20] by inserting catheters in the right radial artery. Radial artery catheterization for sampling preductal blood was chosen for several reasons. First, direct arterial puncture is painful and makes the baby cry, which is known to raise the pulmonary artery pressure and therefore the $\mathrm{R} \rightarrow \mathrm{L}$ shunt. Second, we have had no success with the technique of cannulation of the temporal artery for serial samples using a scalp vein needle. Third, capillary samples may be particularly unreliable for measurement of $\mathrm{paO}_{2}$ [14]. We chose the right radial artery because the left subclavian artery occasionally arises beyond the ductus arteriosus and also because turbulence in the aorta from blood coming out of the ductus may cause some deoxygenated blood to enter a normally situated left subclavian artery. Our technique allowed the blood to be sampled frequently without disturbing the sick newborn baby.

This report presents the results of blood gas estimations on samples obtained simultaneously from the right radial and umbilical arteries in 21 infants with RDS.

\section{Material and Methods}

The babies were studied during a two-year period. Eleven were born in Hammersmith Hospital. Ten were born in outside units and transferred to this center for management. They were all diagnosed clinically as having RDS by exhibiting two or three of the following signs at four hours of age: a respiratory rate of 60 or more, expiratory grunting audible without a stethoscope, and chest retraction. X-rays were taken and were compatible with the clinical diagnosis. Apart from diagnosis of RDS, no selection of cases was carried out other than that imposed by the availability of research staff and technical facilities at the time of admission and diagnosis. The babies were nursed in Oxygenaire Series III incubators at high humidity. The incubator temperature was regulated to maintain the baby's rectal temperature between 35 and $37^{\circ}$. Occasionally, readings were outside that range, usually immediately after admission, when slightly lower values were recorded. The lowest recorded rectal temperature was $34.4^{\circ}$ and the highest, $37.8^{\circ}$. In the latter half of the study, babies were also covered by a radiant heat shield as described by Hey and Mount [11]. An early feeding regime was used [24] and infants were given intravenous therapy only for hypoglycemia (true glucose less than $20 \mathrm{mg} / 100 \mathrm{ml}$ ) or if they were not tolerating oral feeding. Antibiotics were not given routinely but if suspicion of infection existed, patients were treated with penicillin, kanamycin and polymixin.

Umbilical artery catheters were inserted as soon as possible after admission to the Neonatal Ward. FG 5 polyvinyl feeding tubes were inserted to a distance calculated to place the tip of the catheter just below the diaphragm [9]. To sample preductal blood, a FG 2 polyvinyl intravenous catheter of external diameter 
$0.63 \mathrm{~mm}$ was inserted into the right radial artery at the wrist by a cut-down technique. The procedure is particularly easy in very small premature infants who have little subcutaneous fat. The deep fascia was divided and the artery cleaned. The superficial palmar branch may be large and torn at this stage. Gentle pressure over the artery, however, usually staunches the flow and the procedure is not jeopardized. The artery was tied distally with catgut and a small nick made in its wall. Hemorrhage can usually be easily controlled by traction on the distal tie. If this fails, a small bulldog clamp can be applied proximally with good control of bleeding. The catheter was then inserted and not pushed farther than the division of the brachial artery into its ulnar and radial branches. It was held in place with catgut and maintained patent with heparinized saline (10 units/ml). Constant perfusion was not found necessary. The skin was closed with interrupted atraumatic 5-0 black silk sutures. To remove the catheter, it was simply pulled out and pressure applied to the artery for 5 to 10 minutes. The radial artery pulse usually disappeared afterwards but the ulnar pulse became more marked. At no stage before or after the procedure did we observe any signs that circulation to the hand had been compromised. Hemorrhage was never a problem and the infants had no sequelae apart from an absent radial pulse.

Once catheters were in situ, the babies were managed in our conventional way $[8,20]$. Sufficient oxygen was given to maintain $\mathrm{paO}_{2}$ at satisfactory levels and acidosis was corrected by intravenous injections of THAM or sodium bicarbonate. Eight of the babies deteriorated sufficiently to need artificial ventilation [7]; four of them survived.

Blood samples were withdrawn simultaneously from both catheters, the dead spaces of which were filled with heparin (1000 units/ml), into new disposable plastic syringes. The baby's temperature was noted and the oxygen concentration in the incubator measured with a Beckman D2 oxygen analyzer. The blood gases were measured immediately after blood was withdrawn. Levels of $\mathrm{paO}_{2}, \mathrm{pH}$, and $\mathrm{pCO}_{2}$ were measured at $37^{\circ}$ on an IL blood gas apparatus, which was calibrated with gases of known oxygen and $\mathrm{CO}_{2}$ concentrations and buffers of known $\mathrm{pH}$. In calculating $\mathrm{pO}_{2}$, corrections were applied for the difference between the measured $\mathrm{pO}_{2}$ of a gas mixture and the $\mathrm{pO}_{2}$ of blood tonometered with it, a value $3 \%$ lower than that of true $\mathrm{pO}_{2}$ [20]. Temperature corrections for $\mathrm{pCO}_{2}$ and for $\mathrm{pO}_{2}$ less than $200 \mathrm{~mm} \mathrm{Hg}$ were made according to BRADLEY et al. [1]; for $\mathrm{pO}_{2}$ more than $200 \mathrm{~mm} \mathrm{Hg}$, the factors of HeDley-White and LAver [10] were used. The $\mathrm{pH}$ was corrected to body temperature of the subject by the method of Rosenthal [21]. The $\mathrm{R} \rightarrow \mathrm{L}$ shunt was calculated by the method of STRANG and McLeish [26], assuming an A/V oxygen content difference of $4 \mathrm{ml} / 100 \mathrm{ml}$ of blood. It is recognized that, at low environmental oxygen concentrations, there are errors in the calculation of the shunt due to ventilationperfusion imbalances. These errors would tend to increase the calculated size of the shunt and are recognized in the discussion of the results.

\section{Results}

In the 21 babies studied, between one and seventeen simultaneous blood samples were taken to estimate $\mathrm{paO}_{2}, \mathrm{pH}$, and $\mathrm{pCO}_{2}$. In no case was there any significant difference between $\mathrm{pH}$ and $\mathrm{pCO}_{2}$ values obtained from the two sites. Many values for $\mathrm{paO}_{2}$ differed in the radial and umbilical arterial samples, and in all cases the radial value was higher than the umbilical one. There is an error of $\pm 5 \mathrm{~mm} \mathrm{Hg}$ in using the $\mathrm{pO}_{2}$ electrode [6]. We had confirmed this figure in trials in which we had measured the $\mathrm{pO}_{2}$ of tonometered blood; therefore we decided to regard as not significant all $\mathrm{pO}_{2}$ differences of $10 \mathrm{~mm} \mathrm{Hg}$ or less. On this basis, it was possible to divide the babies into two groups.

Group 1. Those babies with no significant $\mathrm{paO}_{2}$ differences (i.e., no pair of blood samples had $\mathrm{paO}_{2}$ values differing by more than $10 \mathrm{~mm} \mathrm{Hg}$ ). There were six such babies (table I).

Group 2. Those babies who sometimes showed paired samples with a radial $\mathrm{paO}_{2}$ value more than $10 \mathrm{~mm}$ $\mathrm{Hg}$ higher than that of the aortic $\mathrm{paO}_{2}$ value. They were divided into three groups.

(a) Six babies who showed occasional radial $\mathrm{paO}_{2}$ more than $10 \mathrm{~mm} \mathrm{Hg}$ higher than values for aortic $\mathrm{paO}_{2}$, but only when aortic $\mathrm{paO}_{2}$ values were more than $140 \mathrm{~mm} \mathrm{Hg}$ (often obtained during a nitrogen washout), never when aortic $\mathrm{paO}_{2}$ values were below this (table II).

(b) Three babies who showed occasional radial $\mathrm{paO}_{2}$ values of more than $10 \mathrm{~mm} \mathrm{Hg}$ higher than aortic $\mathrm{paO}_{2}$ values while on intermittent positive pressure ventilation. These differences were again at $\mathrm{paO}_{2}$ values higher than we usually attempt to maintain our babies (table III).

(c) Six babies who showed occasional radial $\mathrm{paO}_{2}$ values of more than $10 \mathrm{~mm} \mathrm{Hg}$ higher than aortic $\mathrm{paO}_{2}$ at aortic $\mathrm{paO}_{2}$ values of less than $100 \mathrm{~mm} \mathrm{Hg}$ (table IV).

This latter group, 2(c), must be regarded as the most significant since the differences occurred within the range of $\mathrm{paO}_{2}$ at which we normally maintain babies. The records of these six babies were carefully scrutinized to try to identify some common feature that might separate them from the other 15. None was found. Their weights and gestational ages covered a large span from 1260-2930 $\mathrm{g}$ and from 31 weeks to term 
Table I. Group 1. Babies with no recorded differences between radial and aortic $\mathrm{paO}_{2}$

\begin{tabular}{|c|c|c|c|c|c|c|c|c|c|c|}
\hline \multirow[t]{2}{*}{$\begin{array}{l}\text { Baby } \\
\text { No. }\end{array}$} & \multirow{2}{*}{$\begin{array}{c}\text { Birth } \\
\text { weight } \\
\\
\mathrm{g} \\
\end{array}$} & \multirow{2}{*}{$\begin{array}{c}\text { Gesta- } \\
\text { tional } \\
\text { age } \\
\text { weeks } \\
\end{array}$} & \multirow{2}{*}{$\begin{array}{c}\text { Age } \\
\mathrm{h} \\
\end{array}$} & \multirow[t]{2}{*}{$\mathrm{F}_{\mathrm{I}} \mathrm{O}_{2}$} & \multicolumn{2}{|c|}{$\mathrm{paO}_{2}, \mathrm{~mm} \mathrm{Hg}$} & \multicolumn{2}{|c|}{$\begin{array}{l}\% \mathrm{R} \rightarrow \mathrm{L} \text { shunt } \\
\text { calculated }:\end{array}$} & \multirow{2}{*}{$\begin{array}{l}\% \text { shunt } \\
\text { through } \\
\text { ductus }\end{array}$} & \multirow[t]{2}{*}{ Comment } \\
\hline & & & & & Aortic & Radial & Aortic & Radial & & \\
\hline 1 & 1320 & $28^{6} / 7$ & $\begin{array}{l}3 \\
31 / 4 \\
4 \\
83 / 4 \\
131 / 4 \\
16^{3 / 4}\end{array}$ & $\begin{array}{l}0.60 \\
1.00 \\
0.85 \\
0.86 \\
0.80 \\
0.93\end{array}$ & $\begin{array}{r}60 \\
121 \\
105 \\
56 \\
37 \\
60\end{array}$ & $\begin{array}{r}63 \\
117 \\
99 \\
57 \\
37 \\
58\end{array}$ & $\begin{array}{l}35 \\
32 \\
29 \\
42 \\
58 \\
40\end{array}$ & $\begin{array}{l}34 \\
32 \\
29 \\
41 \\
58 \\
41\end{array}$ & $\begin{array}{l}+1 \\
- \\
-1 \\
-1\end{array}$ & $\begin{array}{l}\text { died with } \\
\text { IVH, pneu- } \\
\text { mothorax } \\
\text { and hyaline } \\
\text { membrane } \\
\text { disease }\end{array}$ \\
\hline 2 & 1160 & $31^{4 / 7}$ & $\begin{array}{l}52 \\
531 / 4\end{array}$ & $\begin{array}{l}1.00 \\
1.00\end{array}$ & $\begin{array}{l}15 \\
30\end{array}$ & $\begin{array}{l}16 \\
29\end{array}$ & $\begin{array}{r}>75 \\
68\end{array}$ & $\begin{array}{r}>75 \\
69\end{array}$ & $\overline{-1}$ & $\begin{array}{l}\text { died with } \\
\text { IVH and } \\
\text { hyaline } \\
\text { membrane } \\
\text { disease }\end{array}$ \\
\hline 3 & 1840 & 34 & $\begin{array}{l}171 / 2 \\
193 / 4 \\
211 / 2 \\
303 / 4 \\
403 / 4 \\
44^{3} 3 / 4 \\
561 / 4 \\
653 / 4 \\
69\end{array}$ & $\begin{array}{l}0.88 \\
0.88 \\
0.96 \\
0.92 \\
0.72 \\
0.70 \\
0.94 \\
0.94 \\
0.96\end{array}$ & $\begin{array}{r}78 \\
65 \\
106 \\
91 \\
50 \\
36 \\
60 \\
86 \\
87\end{array}$ & $\begin{array}{r}81 \\
58 \\
100 \\
101 \\
50 \\
36 \\
63 \\
76 \\
96\end{array}$ & $\begin{array}{l}35 \\
39 \\
33 \\
33 \\
44 \\
58 \\
41 \\
35 \\
35\end{array}$ & $\begin{array}{l}34 \\
42 \\
33 \\
32 \\
44 \\
58 \\
40 \\
37 \\
33\end{array}$ & $\begin{array}{l}+1 \\
-3 \\
- \\
+1 \\
- \\
- \\
+1 \\
-2 \\
+2\end{array}$ & $\begin{array}{l}\text { alive and } \\
\text { well }\end{array}$ \\
\hline 4 & 1220 & 29 & $\begin{array}{l}51 / 2 \\
6 \\
7 \\
14 \\
143 / 4 \\
151 / 4\end{array}$ & $\begin{array}{l}0.38 \\
0.20 \\
1.00 \\
0.34 \\
0.20 \\
0.20\end{array}$ & $\begin{array}{r}135 \\
64 \\
326 \\
75 \\
73 \\
49\end{array}$ & $\begin{array}{r}135 \\
61 \\
318 \\
80 \\
75 \\
45\end{array}$ & $\begin{array}{l}10 \\
18 \\
21 \\
21 \\
12 \\
32\end{array}$ & $\begin{array}{l}10 \\
20 \\
21 \\
19 \\
11 \\
36\end{array}$ & $\begin{array}{l}\overline{-2} \\
-\overline{+} \\
+1 \\
-4\end{array}$ & $\begin{array}{l}\text { alive and } \\
\text { well }\end{array}$ \\
\hline 5 & 1600 & 30 & $\begin{array}{l}191 / 2 \\
243 / 4\end{array}$ & $\begin{array}{l}0.92 \\
0.92\end{array}$ & $\begin{array}{l}48 \\
50\end{array}$ & $\begin{array}{l}48 \\
51\end{array}$ & $\begin{array}{l}48 \\
47\end{array}$ & $\begin{array}{l}48 \\
47\end{array}$ & - & $\begin{array}{l}\text { died with } \\
\text { IVH and } \\
\text { hyaline } \\
\text { membrane } \\
\text { disease }\end{array}$ \\
\hline 6 & 2790 & 37 & $\begin{array}{l}5 \\
53 / 4 \\
91 / 2 \\
10^{1 / 2}\end{array}$ & $\begin{array}{l}0.50 \\
1.00 \\
0.68 \\
0.74\end{array}$ & $\begin{array}{r}71 \\
117 \\
79 \\
59\end{array}$ & $\begin{array}{r}71 \\
115 \\
76 \\
61\end{array}$ & $\begin{array}{l}28 \\
32 \\
29 \\
38\end{array}$ & $\begin{array}{l}28 \\
32 \\
30 \\
37\end{array}$ & $\begin{array}{l}- \\
-1 \\
+1\end{array}$ & $\begin{array}{l}\text { alive and } \\
\text { well }\end{array}$ \\
\hline
\end{tabular}

$\mathrm{F}_{\mathrm{I}} \mathrm{O}_{2}=$ Inspired oxygen $\quad 1.00(\mathrm{I})=100 \%$ oxygen by intermittent positive pressure ventilation $\mathrm{IVH}=$ Intraventricular hemorrhage. 
Table II. Group 2(a). Babies with radial $\mathrm{paO}_{2}>10 \mathrm{~mm} \mathrm{Hg}$ higher than aortic $\mathrm{paO}_{2}$ at high $\mathrm{paO}_{2}$ levels

\begin{tabular}{|c|c|c|c|c|c|c|c|c|c|c|c|}
\hline \multirow[t]{2}{*}{$\begin{array}{l}\text { Baby } \\
\text { No. }\end{array}$} & \multirow{2}{*}{$\begin{array}{c}\text { Birth } \\
\text { weight } \\
\\
\mathrm{g} \\
\end{array}$} & \multirow{2}{*}{$\begin{array}{c}\text { Gesta- } \\
\text { tional } \\
\text { age } \\
\text { weeks }\end{array}$} & \multirow{2}{*}{$\begin{array}{c}\text { Age } \\
\\
\mathrm{h} \\
\end{array}$} & \multirow[t]{2}{*}{$\mathrm{F}_{\mathrm{I}} \mathrm{O}_{2}$} & \multicolumn{2}{|c|}{$\mathrm{paO}_{2}, \mathrm{~mm} \mathrm{Hg}$} & \multicolumn{2}{|c|}{$\begin{array}{l}\% \mathrm{R} \rightarrow \mathrm{L} \text { shunt } \\
\text { calculated: }\end{array}$} & \multirow{2}{*}{$\begin{array}{c}\% \text { shunt } \\
\text { through } \\
\text { ductus }\end{array}$} & \multirow{2}{*}{$\begin{array}{c}\text { Ductus } \\
\text { shunt } \\
\text { Total } \\
\text { shunt } \\
\%\end{array}$} & \multirow[t]{2}{*}{ Comment } \\
\hline & & & & & Aortic & Radial & Aortic & Radial & & & \\
\hline 10 & 1760 & $31 \frac{1 / 2}{2}$ & $\begin{array}{r}51 / 2 \\
71 / 4 \\
111 / 4 \\
153 / 4 \\
211 / 2 \\
381 / 2 \\
441 / 2\end{array}$ & $\begin{array}{l}0.72 \\
0.48 \\
0.56 \\
0.56 \\
0.42 \\
0.40 \\
0.40\end{array}$ & $\begin{array}{r}161 \\
65 \\
83 \\
128 \\
97 \\
80 \\
76\end{array}$ & $\begin{array}{c}176^{*} \\
63 \\
83 \\
132 \\
101 \\
84 \\
71\end{array}$ & $\begin{array}{l}21 \\
30 \\
26 \\
19 \\
17 \\
21 \\
23\end{array}$ & $\begin{array}{l}20 \\
29 \\
26 \\
18 \\
16 \\
19 \\
24\end{array}$ & $\begin{array}{l}+1 \\
+1 \\
+1 \\
+1 \\
+2 \\
-1\end{array}$ & 4.8 & $\begin{array}{l}\text { alive and } \\
\text { well }\end{array}$ \\
\hline 11 & 1400 & 29 & 120 & 0.95 & 143 & $188 *$ & 29 & 26 & +3 & 10.4 & $\begin{array}{l}\text { alive and } \\
\text { well }\end{array}$ \\
\hline 12 & 1800 & 37 & $\begin{array}{l}31 / 2 \\
41 / 4 \\
43 / 4\end{array}$ & $\begin{array}{l}0.94 \\
0.52 \\
0.34\end{array}$ & $\begin{array}{l}283 \\
183 \\
118\end{array}$ & $\begin{array}{l}343 * \\
179 \\
113\end{array}$ & $\begin{array}{l}21 \\
12 \\
10\end{array}$ & $\begin{array}{l}17 \\
13 \\
11\end{array}$ & $\begin{array}{l}+4 \\
-1 \\
--1\end{array}$ & 19.1 & $\begin{array}{l}\text { alive and } \\
\text { well }\end{array}$ \\
\hline 13 & 1300 & 32 & $\begin{array}{r}31 / 4 \\
33 / 4 \\
163 / 4 \\
211 / 4 \\
213 / 4 \\
\end{array}$ & $\begin{array}{l}0.30 \\
1.00 \\
0.28 \\
0.25 \\
1.00 \\
\end{array}$ & $\begin{array}{r}99 \\
298 \\
97 \\
78 \\
326 \\
\end{array}$ & $\begin{array}{c}96 \\
327 * \\
98 \\
76 \\
369 * \\
\end{array}$ & $\begin{array}{l}10 \\
22 \\
10 \\
14 \\
21 \\
\end{array}$ & $\begin{array}{l}10 \\
20 \\
10 \\
16 \\
19 \\
\end{array}$ & $\begin{array}{l}+2 \\
-2 \\
+2 \\
\end{array}$ & 9.1 & $\begin{array}{l}\text { alive and } \\
\text { well }\end{array}$ \\
\hline 14 & 1500 & 32 & $\begin{array}{l}41 / 2 \\
6 \\
19 \\
22 \\
221 / 4 \\
\end{array}$ & $\begin{array}{l}0.74 \\
0.56 \\
0.30 \\
0.40 \\
1.00 \\
\end{array}$ & $\begin{array}{r}170 \\
120 \\
92 \\
96 \\
220 \\
\end{array}$ & $\begin{array}{r}190 * \\
116 \\
90 \\
97 \\
230 \\
\end{array}$ & $\begin{array}{l}21 \\
20 \\
16 \\
17 \\
26 \\
\end{array}$ & $\begin{array}{l}19 \\
20 \\
16 \\
17 \\
26 \\
\end{array}$ & +2 & 9.55 & $\begin{array}{l}\text { alive and } \\
\text { well }\end{array}$ \\
\hline 15 & 2260 & 37 & $\begin{array}{l}91 / 2 \\
93 / 4 \\
101 / 2 \\
26 \\
321 / 2 \\
371 / 2 \\
47 \\
521 / 2 \\
561 / 2 \\
611 / 2 \\
711 / 2 \\
751 / 2 \\
1271 / 2 \\
128 \\
1461 / 2 \\
147\end{array}$ & $\begin{array}{l}0.48 \\
1.00 \\
0.58 \\
0.90 \\
0.94 \\
0.90 \\
0.92 \\
0.90 \\
0.92 \\
0.90 \\
0.97 \\
0.97 \\
0.64 \\
1.00 \\
0.38 \\
1.00\end{array}$ & $\begin{array}{r}61 \\
156 \\
61 \\
72 \\
66 \\
49 \\
47 \\
49 \\
49 \\
50 \\
70 \\
63 \\
76 \\
209 \\
53 \\
177\end{array}$ & $\begin{array}{c}59 \\
182 * \\
61 \\
71 \\
63 \\
55 \\
49 \\
49 \\
50 \\
53 \\
67 \\
68 \\
75 \\
224 \\
58 \\
182\end{array}$ & $\begin{array}{l}31 \\
30 \\
34 \\
37 \\
40 \\
47 \\
48 \\
47 \\
47 \\
47 \\
38 \\
40 \\
30 \\
26 \\
35 \\
29\end{array}$ & $\begin{array}{l}32 \\
28 \\
34 \\
38 \\
41 \\
43.5 \\
47 \\
47 \\
47 \\
45 \\
39 \\
39 \\
30 \\
25 \\
29 \\
29\end{array}$ & $\begin{array}{l}-1 \\
+2 \\
-1 \\
-1 \\
+3.5 \\
+1 \\
\\
+2 \\
-1 \\
+1 \\
+1 \\
+6\end{array}$ & $\begin{array}{r}3.8 \\
17.2\end{array}$ & $\begin{array}{l}\text { alive and } \\
\text { well }\end{array}$ \\
\hline
\end{tabular}

See table I for abbreviations.

* Significant differences between aortic and radial $\mathrm{paO}_{2}$.

1 Calculated when the \% of the cardiac output passing through the ductus was $>5 \%$ and when the radial $\mathrm{paO}_{2}$ was $>10 \mathrm{~mm} \mathrm{Hg}$ higher than the aortic $\mathrm{paO}_{2}$. 
Table III. Group 2(b). Babies with radial $\mathrm{paO}_{2}>10 \mathrm{~mm} \mathrm{Hg}$ higher than aortic $\mathrm{paO}_{2}$ when being artificially ventilated

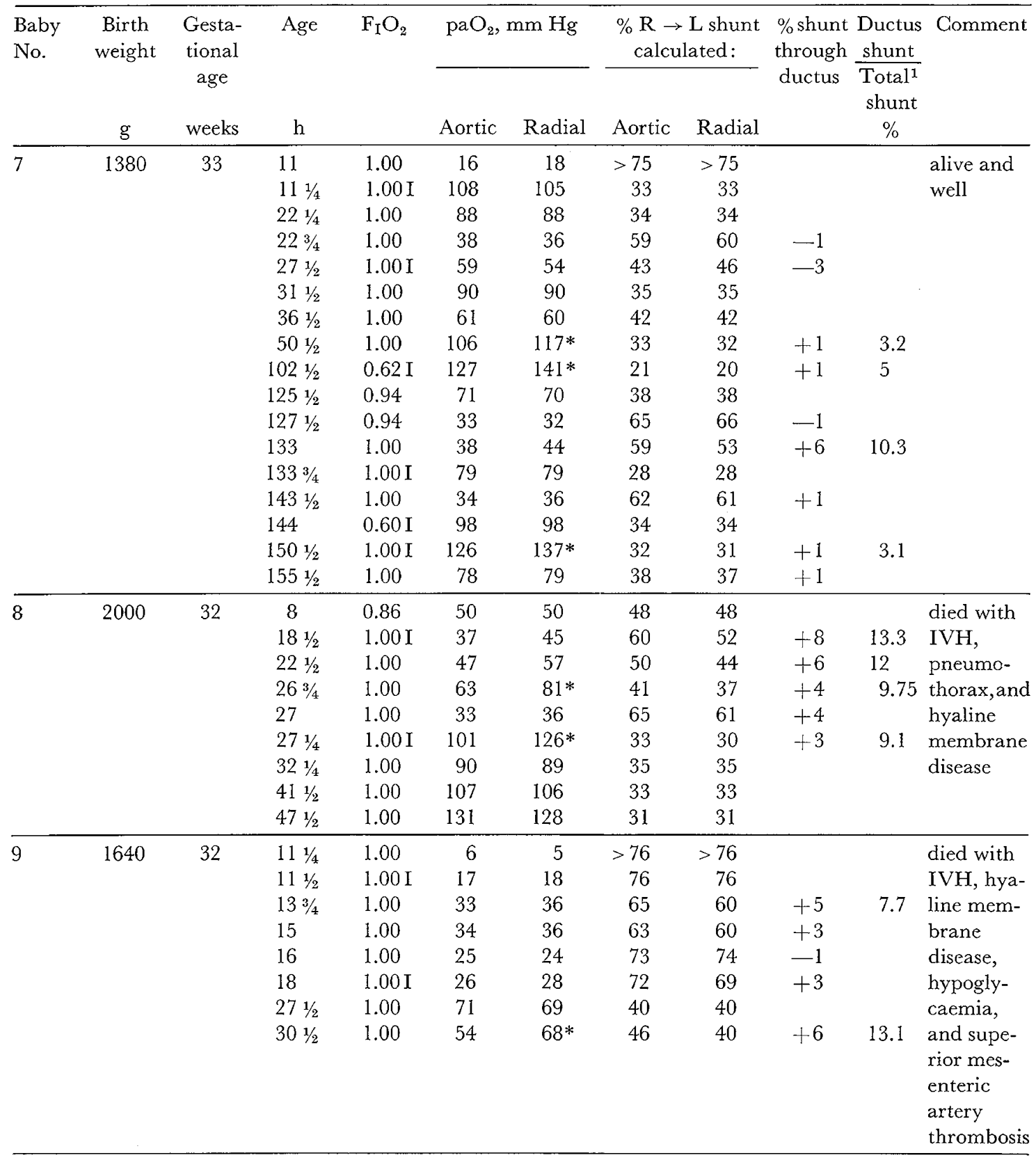

See tables I and II for abbreviations.

* Significant differences between aortic and radial $\mathrm{paO}_{2}$.

${ }^{1}$ Calculated when the $\%$ of the cardiac output passing through the ductus was $>5 \%$ and when the radial $\mathrm{paO}_{2}$ was $>10 \mathrm{~mm} \mathrm{Hg}$ higher than the aortic $\mathrm{paO}_{2}$. 
Table IV. Group 2(c). Babies with radial $\mathrm{paO}_{2}>10 \mathrm{~mm} \mathrm{Hg}$ higher than aortic $\mathrm{paO}_{2}$ at normal aortic $\mathrm{paO}_{2}$ values

\begin{tabular}{|c|c|c|c|c|c|c|c|c|c|c|c|}
\hline \multirow[t]{2}{*}{$\begin{array}{l}\text { Baby } \\
\text { No. }\end{array}$} & \multirow{2}{*}{$\begin{array}{c}\text { Birth } \\
\text { weight } \\
\\
\mathrm{g} \\
\end{array}$} & \multirow{2}{*}{$\begin{array}{c}\text { Gesta- } \\
\text { tional } \\
\text { age } \\
\text { weeks }\end{array}$} & \multirow{2}{*}{$\begin{array}{c}\text { Age } \\
\text { h } \\
\end{array}$} & \multirow[t]{2}{*}{$\mathrm{F}_{\mathrm{I}} \mathrm{O}_{2}$} & \multicolumn{2}{|c|}{$\mathrm{paO}_{2}, \mathrm{~mm} \mathrm{Hg}$} & \multicolumn{2}{|c|}{$\begin{array}{c}\% \mathrm{R} \rightarrow \mathrm{L} \text { shunt } \\
\text { calculated: }\end{array}$} & \multirow{2}{*}{$\begin{array}{l}\text { \% shunt } \\
\text { through } \\
\text { ductus }\end{array}$} & \multirow{2}{*}{$\begin{array}{c}\begin{array}{c}\text { Ductus } \\
\text { shunt }\end{array} \\
\text { Total }{ }^{1} \\
\text { shunt } \\
\%\end{array}$} & \multirow{2}{*}{ Comment } \\
\hline & & & & & Aortic & Radial & Aortic & Radial & & & \\
\hline \multirow[t]{6}{*}{16} & 2930 & 40 & 12 & 0.95 & 30 & 34 & 67 & 62 & +5 & 7.5 & died at $194 \mathrm{~h}$ \\
\hline & & & $123 / 4$ & 0.95 & 41 & $53 *$ & 55 & 46 & +9 & 16.3 & with airways \\
\hline & & & 17 & 0.94 & 35 & 41 & 61 & 54 & +7 & 11.5 & obstruction \\
\hline & & & 23 & 0.94 & 42 & 47 & 54 & 55 & -1 & & from re- \\
\hline & & & 26 & 0.94 & 29 & 32 & 68 & 66 & +2 & & tained secre- \\
\hline & & & 28 & 0.96 & 30 & 35 & 68 & 61 & +7 & 10.3 & $\begin{array}{l}\text { tions on ven- } \\
\text { tilator, IVH, } \\
\text { ASD present }\end{array}$ \\
\hline \multirow[t]{7}{*}{$\overline{17}$} & 2060 & $33^{1 / 7}$ & 4 & 0.84 & 65 & $77 *$ & 38 & 34 & +4 & 10.5 & alive and \\
\hline & & & 7 & 0.82 & 41 & 48 & 54 & 47 & +7 & 13 & well \\
\hline & & & $10^{3 / 4}$ & 0.93 & 51 & $64^{*}$ & 47 & 40 & +7 & 14.9 & subse- \\
\hline & & & $111 / 2$ & 0.94 & 89 & 96 & 34 & 33 & +1 & & quently \\
\hline & & & $213 / 4$ & 0.96 & 64 & 63 & 41 & 41 & & & had to be \\
\hline & & & $221 / 4$ & 0.94 & 54 & 53 & 45 & 46 & -1 & & ventilated \\
\hline & & & $271 / 4$ & 0.90 & 83 & 78 & 34 & 35 & -1 & & for 2 days \\
\hline \multirow[t]{6}{*}{18} & 2100 & 35 & 9 & 0.32 & 56 & $70^{*}$ & 30 & 22 & +8 & 26.6 & alive and \\
\hline & & & $91 / 4$ & 1.00 & 250 & $278 *$ & 25 & 23 & +2 & 8 & well \\
\hline & & & $131 / 4$ & 0.52 & 136 & 145 & 16 & 15 & +1 & & \\
\hline & & & $163 / 4$ & 0.52 & 73 & 72 & 27 & 28 & -1 & & \\
\hline & & & $203 / 4$ & 0.56 & 82 & 78 & 26 & 27 & -1 & & \\
\hline & & & $21 \frac{1}{4}$ & 1.00 & 231 & 226 & 25 & 25 & & & \\
\hline \multirow[t]{6}{*}{$\overline{19}$} & 1260 & 31 & $21 / 4$ & 0.46 & 57 & $93^{*}$ & 33 & 20 & +13 & 39.3 & alive and \\
\hline & & & $23 / 4$ & 1.00 & 195 & $290 *$ & 27 & 22 & +5 & 18.5 & well \\
\hline & & & $33 / 4$ & 0.54 & 74 & $120 *$ & 28 & 19 & +9 & 32.2 & \\
\hline & & & $131 / 4$ & 0.36 & 61 & 59 & 28 & 29 & -1 & & \\
\hline & & & $143 / 4$ & 1.00 & 173 & $262 *$ & 29 & 24 & +5 & 17.3 & \\
\hline & & & $251 / 2$ & 0.44 & 111 & 109 & 16 & 16 & & & \\
\hline \multirow[t]{8}{*}{20} & 1800 & $33^{4} / 7$ & $363 / 4$ & 0.94 & 78 & 73 & 36 & 38 & -2 & & alive and \\
\hline & & & $451 / 2$ & 0.94 & 76 & $97 *$ & 37 & 32 & +5 & 13.5 & well had Ap- \\
\hline & & & 46 & 0.94 & 82 & $114 *$ & 35 & 31 & +4 & $11.4 \xi$ & gar of 0 at 1 \\
\hline & & & $561 / 2$ & 0.96 & 131 & 125 & 30 & 31 & -1 & & min and arti- \\
\hline & & & $643 / 4$ & 0.94 & 57 & 59 & 43 & 42 & +1 & & ficially ventilat- \\
\hline & & & $68 x / 2$ & 0.94 & 67 & 65 & 39 & 40 & -1 & & ed for 1 st $20 \mathrm{~h}$ of \\
\hline & & & 83 & 0.94 & 52 & 56 & 46 & 44 & +2 & & life subsequent- \\
\hline & & & 89 & 0.94 & 64 & 64 & 40 & 40 & & & ly thrived \\
\hline \multirow[t]{6}{*}{21} & 1730 & $356 / 7$ & $263 / 4$ & 0.60 & 127 & 122 & 21 & 21 & & & alive and well \\
\hline & & & $271 / 4$ & 1.00 & 238 & 238 & 26 & 26 & & & subsequent- \\
\hline & & & 29 & 0.64 & 133 & 132 & 21 & 21 & & & ly had to be \\
\hline & & & $401 / 2$ & 0.88 & 64 & $108 *$ & 39 & 30 & +9 & 23.1 & artificially \\
\hline & & & 41 & 0.82 & 48 & 53 & 47 & 44 & +3 & 6.3 & ventilated \\
\hline & & & 43 & 0.86 & 147 & $161 *$ & 27 & 25 & +2 & $7.4 \quad 1$ & for $60 \mathrm{~h}$ \\
\hline
\end{tabular}

See tables I and II for abbreviations.

* Significant differences between aortic and radial $\mathrm{paO}_{2}$.

${ }^{1}$ Calculated when the $\%$ of the cardiac output passing through the ductus was $>5 \%$ and when the radial $\mathrm{paO}_{2}$ was $>10 \mathrm{~mm} \mathrm{Hg}$ higher than the aortic $\mathrm{paO}_{2}$.

$\mathrm{ASD}=$ Atrial septal defect. 


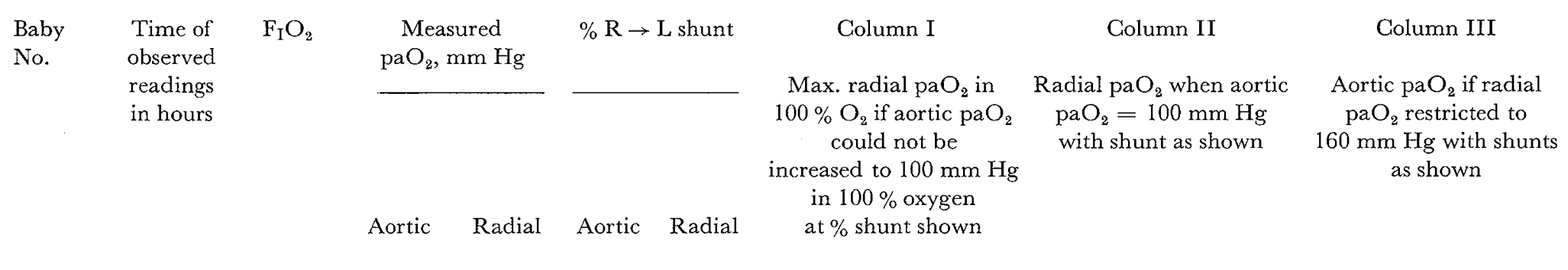

\begin{tabular}{|c|c|c|c|c|c|c|c|c|c|}
\hline 16 & $123 / 4$ & 0.95 & 41 & 53 & 55 & 46 & 53 & - & - \\
\hline 17 & $\begin{array}{c}4 \\
103 / 4\end{array}$ & $\begin{array}{l}0.84 \\
0.84\end{array}$ & $\begin{array}{l}65 \\
51\end{array}$ & $\begin{array}{l}77 \\
64\end{array}$ & $\begin{array}{l}38 \\
47\end{array}$ & $\begin{array}{l}34 \\
40\end{array}$ & $\begin{array}{l}94 \\
67\end{array}$ & - & - \\
\hline 18 & $\begin{array}{l}9 \\
9^{1 / 4}\end{array}$ & $\begin{array}{l}0.32 \\
1.00\end{array}$ & $\begin{array}{r}56 \\
250\end{array}$ & $\begin{array}{r}70 \\
278\end{array}$ & $\begin{array}{l}30 \\
25\end{array}$ & $\begin{array}{l}22 \\
23\end{array}$ & - & $\begin{array}{l}220 \\
120\end{array}$ & $\begin{array}{r}80 \\
135\end{array}$ \\
\hline 19 & $\begin{array}{r}21 / 4 \\
23 / 4 \\
33 / 4 \\
143 / 4\end{array}$ & $\begin{array}{l}0.46 \\
1.00 \\
0.54 \\
1.00\end{array}$ & $\begin{array}{r}57 \\
195 \\
74 \\
173\end{array}$ & $\begin{array}{r}93 \\
290 \\
120 \\
262\end{array}$ & $\begin{array}{l}33 \\
27 \\
28 \\
29\end{array}$ & $\begin{array}{l}20 \\
22 \\
19 \\
24\end{array}$ & $\begin{array}{l}- \\
- \\
-\end{array}$ & $\begin{array}{l}320 \\
160 \\
210 \\
160\end{array}$ & $\begin{array}{r}60 \\
100 \\
80 \\
100\end{array}$ \\
\hline 20 & $\begin{array}{l}36 \quad 3 / 4 \\
451 / 2\end{array}$ & $\begin{array}{l}0.94 \\
0.94\end{array}$ & $\begin{array}{l}76 \\
82\end{array}$ & $\begin{array}{r}97 \\
114\end{array}$ & $\begin{array}{l}37 \\
35\end{array}$ & $\begin{array}{l}32 \\
31\end{array}$ & $\begin{array}{l}120 \\
130\end{array}$ & - & - \\
\hline 21 & $\begin{array}{l}401 / 2 \\
431 / 2\end{array}$ & $\begin{array}{l}0.88 \\
0.86\end{array}$ & $\begin{array}{r}64 \\
147\end{array}$ & $\begin{array}{l}108 \\
161\end{array}$ & $\begin{array}{l}39 \\
27\end{array}$ & $\begin{array}{l}30 \\
25\end{array}$ & $\begin{array}{r}140 \\
-\end{array}$ & $\overline{120}$ & $\overline{135}$ \\
\hline
\end{tabular}


respectively. Four babies had severe respiratory distress and eventually had to be artificially ventilated (the discrepant $\mathrm{paO}_{2}$ values were found while the infants were breathing spontaneously), but only one died. The other two babies were mildly affected. We have details of the state at birth in only three of the six babies (Nos. 17, 19 and 20), those born at Hammersmith Hospital. Baby No. 20 scored 0 at 1 minute (Apgar), whereas the other two were in good condition. None was overtly infected at the time of sampling. No baby had any cardiac murmur at the time of sampling and none of the five survivors had any clinical evidence of congenital heart disease, although Baby No. 16 was found to have a small ASD at postmortem examination. It should be noted that the recordings of radial $\mathrm{paO}_{2}$ more than $10 \mathrm{~mm} \mathrm{Hg}$ higher than aortic $\mathrm{paO}_{2}$ were not persistent throughout the course of the baby's illness. In four of the six babies who showed high $\mathrm{paO}_{2}$ differences, the relevant samples were taken during the first 24 hours of life. Subsequent samples showed no significant differences between radial and aortic oxygen tension. The other two babies had high $\mathrm{paO}_{2}$ differences during the second day of life, when previous samples in Baby No.21 and subsequent samples in Baby No. 20 showed no differences.

\section{Discussion}

In previous years it had been our aim to maintain the aortic $\mathrm{paO}_{2}$ at approximately $100 \mathrm{~mm} \mathrm{Hg}$ and always less than $160 \mathrm{~mm} \mathrm{Hg}$ to prevent retrolental fibroplasia [20]. It would appear from the present study that the right radial and hence the carotid and retinal $\mathrm{paO}_{2}$ can be significantly greater than the lower abdominal aorta $\mathrm{paO}_{2}$ sampled via an umbilical arterial catheter.

Assuming a retinal $\mathrm{paO}_{2}$ of $160 \mathrm{~mm} \mathrm{Hg}$ as the maximum safe value [20], what is the highest safe abdominal aortic $\mathrm{paO}_{2}$ ? It is clear from our data that the safe level of aortic $\mathrm{paO}_{2}$ cannot be deduced accurately; however, a reasonable approximation can be derived by calculating the $\mathrm{R} \rightarrow \mathrm{L}$ shunts by the method of Strang and MaLeish [26].

The shunt calculation on the aortic $\mathrm{paO}_{2}$ value will give the total $\mathrm{R} \rightarrow \mathrm{L}$ shunt through the foramen ovale, ductus arteriosus, and the lungs; the shunt calculation on radial $\mathrm{paO}_{2}$ blood will give the $\mathrm{R} \rightarrow \mathrm{L}$ shunt through the foramen ovale and lungs only. The differences between these values will be the percentage of the cardiac output passing $\mathrm{R} \rightarrow \mathrm{L}$ through the ductus arteriosus.

Tables I, II, and III show that in the babies of Groups 1, 2(a) and 2(b), less than $5 \%$ of the cardiac output passed from right to left through the ductus arteriosus-the only exception being a total of six observations on Babies Nos. 7, 8, 9 and 15, in whom the
$\mathrm{paO}_{2}$ was very low in $100 \%$ oxygen and in whom there was no problem of oxygen toxicity to the retina. It should be noted that at low $\mathrm{paO}_{2}$ levels, small differences in observed values, lying within the limits of experimental error, will cause large differences in the calculated shunt. Furthermore, the proportion of the total right to left shunt passing through the ductus exceeded $10 \%$ only on these occasions and on one other occasion, in Baby No. 12, in whom the total shunt was very small.

In contrast, the babies in Group 2(c) sometimes showed $\mathrm{R} \rightarrow \mathrm{L}$ shunts through the ductus arteriosus of more than $5 \%$ of the cardiac output at $\mathrm{paO}_{2}$ levels within the normal range (table IV). The proportion of the total right to left shunt passing through the ductus often exceeded $15 \%$.

The shunt calculations on observed values of radial and aortic $\mathrm{paO}_{2}$ having been made, the radial $\mathrm{paO}_{2}$ was calculated for an aortic $\mathrm{paO}_{2}$ of $100 \mathrm{~mm} \mathrm{Hg}$ (table $\mathrm{V}$, column II) and aortic $\mathrm{paO}_{2}$ was calculated for a radial $\mathrm{paO}_{2}$ of $160 \mathrm{~mm} \mathrm{Hg}$ (table $\mathrm{V}$, column III). It is assumed that the proportion of cardiac output shunted through the ductus arteriosus will remain constant at varying levels of $\mathrm{paO}_{2}$. This assumption is no doubt incorrect but will err on the side of safety, since there is much evidence to suggest that ductus flows are decreased by raising $\mathrm{paO}_{2}[1,18]$.

It will be noted that in Babies Nos. 16, 17, 20 and 21 (first reading), it was not possible to raise the aortic $\mathrm{paO}_{2}$ to $100 \mathrm{~mm} \mathrm{Hg}$, the level at which we wished to maintain the babies (table V, column I). In Babies Nos. 18 and 19, however, high radial $\mathrm{paO}_{2}$ values, greater than the 'danger' level of $160 \mathrm{~mm} \mathrm{Hg}$, would theoretically have been possible in the presence of an aortic $\mathrm{paO}_{2}$ of $100 \mathrm{~mm} \mathrm{Hg}$ (table V, column II) ; conversely, a radial $\mathrm{paO}_{2}$ of $160 \mathrm{~mm} \mathrm{Hg}$ could have been achieved at aortic $\mathrm{paO}_{2}$ values of 80,60 , and $80 \mathrm{~mm} \mathrm{Hg}$ (table V, column III); however, both Babies Nos. 18 and 19 were in low oxygen concentrations at the time these samples were taken. At low ambient oxygen concentrations, and hence at low alveolar $\mathrm{paO}_{2}$ tensions, there are considerably greater errors in the shunt calculations (due to ventilation-perfusion imbalances) than when the subject is breathing $80 \%$ oxygen or more. Hence the calculated shunts in Babies Nos. 18 and 19 while breathing air with comparatively low oxygen concentrations are probably falsely high. Both babies were exposed to $100 \%$ oxygen immediately after the readings, which showed large shunt differences in low environmental oxygen concentrations; in both cases, the anticipated reduction in total shunt took place. The proportion of the shunt passing $\mathrm{R} \rightarrow \mathrm{L}$ through the ductus arteriosus also decreased, indicating a constriction of the ductus arteriosus at the high arterial oxygen tension. The values recorded in $100 \%$ 
oxygen suggested that the retinal $\mathrm{paO}_{2}$ would be unlikely to exceed the 'danger' level of $160 \mathrm{~mm} \mathrm{Hg}$ in an ambient oxygen concentration necessary to raise the aortic $\mathrm{paO}_{2}$ to $100 \mathrm{~mm} \mathrm{Hg}$, if the shunt remained the same.

It has been pointed out that the values for radial $\mathrm{paO}_{2}$ of more than $10 \mathrm{~mm} \mathrm{Hg}$ higher than aortic $\mathrm{paO}_{2}$ did not persist throughout the course of the illness. When present, these usually occurred during the first 24 hours of life. This finding agrees with the greater incidence of bidirectional shunting and higher pulmonary artery pressure found at this time. Presumably, comparatively minor changes in pulmonary artery pressure or systemic blood pressure can alter the hemodynamics at the level of the ductus arteriosus and cause large $\mathrm{R} \rightarrow \mathrm{L}$ shunts of varying size to occur there. The babies in our series were not crying at the time of sampling and this is not the explanation of the variable shunts.

In babies in Group 2(a), the radial $\mathrm{paO}_{2}$ values of more than $10 \mathrm{~mm} \mathrm{Hg}$ higher than aortic $\mathrm{paO}_{2}$ values that were recorded at high arterial oxygen tensions in high environmental oxygen concentrations are merely an indication that at these $\mathrm{paO}_{2}$ values, very small differences in the $\mathrm{R} \rightarrow \mathrm{L}$ shunt will give big $\mathrm{paO}_{2}$ differences (table II).

Babies who are artificially ventilated by positive pressure ventilation have an elevated pulmonary artery pressure. In Group 2(b), three of the babies on IPPR showed small but significant $\mathrm{paO}_{2}$ differences between radial artery and aorta (table III). The considerable fluctuations in oxygen tension and acid base status found in these children may well have played a part in the vascular instability predisposing to $\mathrm{R} \rightarrow \mathrm{L}$ ductus shunts. Babies in Groups 2(a) and 2(b) are therefore of less clinical significance than those in Group 2(c).

The major clinical implication of these findings is that the measurement of aortic $\mathrm{paO}_{2}$ values via an umbilical arterial catheter may not give a true indication of the retinal arterial $\mathrm{pO}_{2}$. From our figures, however, it would seem very unlikely that retinal artery $\mathrm{pO}_{2}$ would exceed $160 \mathrm{~mm} \mathrm{Hg}$ if the umbilical arterial $\mathrm{pO}_{2}$ never exceeded $100 \mathrm{~mm} \mathrm{Hg}$. The inspired oxygen concentrations required to reach these higher arterial $\mathrm{pO}_{2}$ values will lower the pulmonary artery pressure and cause constriction of the ductus arteriosus, thereby decreasing the shunt through it. It seems reasonable, therefore, to try to maintain the umbilical arterial $\mathrm{pO}_{2}$ at about $75 \mathrm{~mm} \mathrm{Hg}$, the normal value in the premature newborn during the first 24 hours of life, and not let it exceed $100 \mathrm{~mm} \mathrm{Hg}$. If this rule is adhered to, only in a very exceptional case, such as that reported by CHU et al. [3], would the retinal artery $\mathrm{pO}_{2}$ reach levels likely to cause retrolental fibroplasia.

\section{Summary}

The technique of radial artery catheterization is described. Fifteen of 21 babies with respiratory distress syndrome had $\mathrm{paO}_{2}$ values sometimes differing by more than $10 \mathrm{~mm} \mathrm{Hg}$ when simultaneous pre- and post-ductal samples were analyzed. In only six was the difference of any clinical significance. The maximum safe abdominal aorta (and hence umbilical) $\mathrm{paO}_{2}$ is $100 \mathrm{~mm} \mathrm{Hg}$.

\section{References and Notes}

1. Bradley, A.F.; Stupfel, M. and Severingmaus, J.W.: The effect of temperature on the $\mathrm{pCO}_{2}$ and $\mathrm{pO}_{2}$ of blood in vitro. J. appl. Physiol. 9: 201 (1956).

2. Gassin, S.; Dawes, G. A.; Mott, J. C. ; Ross, B. B. and Strang, L.B.: The vascular resistance of the foetal and newly ventilated lung of the lamb. J. Physiol. 171: 61 (1964).

3. Cexu, J.; Clements, J.A.; Cotton, E. K.; Klaus, M.H.; Sweet, A.Y. and Tooley, W.H.: Neonatal pulmonary ischaemia. Part I. Clinical and physiological studies. Pediatrics 40: 709 (1967).

4. Eldridge, F. L.; Hultgran, H.N. and Wigmore, M.E. : The physiologic closure of the ductus arteriosus in the newborn infant. J.clin. Invest. 34: 987 (1955).

5. Emmanouilides, G. G.; Moss, A.J.; Duffie, E.R. and Adams, F.H.: Pulmonary arterial pressure changes in human newborn infants from birth to three days of age. J. Pediat. 65: 327 (1964).

6. Flenley, D.C.; Mrllar, J.S. and Rees, H.A.: Accuracy of oxygen and carbon dioxide electrodes. Brit. med.J. ii: 349 (1967).

7. Grausz, J.P.; Watt, N.L. and Becket, A.J.: A new positive-pressure respirator for newborns. Lancet ii: 499 (1967).

8. Gupta, J.M.; Dahlenburg, G.W. and Davis, J.A.: Changes in blood gas tensions following administration of Amine Buffer THAM to infants with respiratory distress syndrome. Arch.Dis. Childh. 42: 416 (1967).

9. Gupta, J.M.; Roberton, N.R.C. and WiggleswORTH, J.S.: Umbilical arterial catheterization in the newborn. Arch. Dis. Childh. 43: 382 (1968).

10. Hedley-White, J. and Laver, M.B.: Oxygen solubility in blood and temperature correction factors for $\mathrm{pO}_{2}$. J.appl. Physiol. 19: 901 (1964).

11. Hey, E. and Mount, L.: Temperature control in incubators. Lancet ii: 203 (1966).

12. James, L.S.; Burnard, E.D. and Rowe, R.D.: Abnormal shunting through the foramen ovale after birth. Amer.J. Dis. Child. 102: 550 (1961). 
13. James, L. S. and Rowe, R.D.: The pattern of response of pulmonary and systemic arterial pressures in newborn and older infants to short periods of hypoxia. J. Pediat. 51: 5 (1957).

14. Koch, G. and Wendel, H.: Comparison of $\mathrm{pH}$, carbon dioxide tension, standard bicarbonate and oxygen tension in capillary blood and in arterial blood during the neonatal period. Acta paediat. scand. 56: 10 (1967).

15. Moss, A.J.; Emmanoullides, G. C.; Adams, F.H. and CHUANG, K. : Response of the ductus arteriosus and pulmonary and systemic arterial pressure to changes in oxygen environment in newborn infants. Pediatrics 33: 937 (1964).

16. Moss, A.J.; Emmanouilides, G.C. and Duffie, E.R.: Closure of the ductus arteriosus in the newborn infant. Pediatrics 32: 25 (1963).

17. Nelson, N.H.; Prod'hom, L.S.; Gherry, R.B.; Lipsitz, P.L. and Sмiтh, C.A.: Pulmonary function in the newborn infant-the alveolar arterial oxygen gradient. J.appl. Physiol. 18: 534 (1963).

18. Oliver, T.K.; Demis, J.A. and Bates, G.D.: Serial blood gas tensions and acid base balance during the first hour of life in human infants. Acta paediat. scand. 50: 346 (1961).

19. Prec, K. J. and Cassels, D. E. : Dye dilution curves and cardiac output in newborn infants. Circulation II: 789 (1955).

20. Roberton, N.R. G.; Gupta, J. M.; Dahlenburg, G.W. and Trzard, J. P. M. : Oxygen therapy in the newborn. Lancet $i: 1323$ (1968).

21. Rosenthal, T.B.: The effect of temperature on the $\mathrm{pH}$ of blood and plasma in vitro. J. biol. Chem. 173: 25 (1948).
22. Rudolph, A. M.; Drorbaugh, J.E.; Auld, P.A.; Rudolph, A.J.; Nadas, A.F.; Smith, C.A. and Hubbell, J.P.: Studies on the circulation in the neonatal period: The circulation in the respiratory distress syndrome. Pediatrics 27: 551 (1961).

23. Rudolph, A.M. and YuAN, S.: Response of the pulmonary vasculature to hypoxia and $\mathrm{H}^{+}$ion concentration changes. J. clin. Invest.45:399 (1966).

24. Smallpeice, V. and Davies, P.A.: Immediate feeding of premature infants with undiluted breast milk. Lancet ii: 1349 (1964).

25. Stahlman, M.: Treatment of cardiovascular disorders of the newborn. Pediat. Clin. N. Amer. 11: 364 (1964).

26. Strang, L.B. and MacLeish, M.H.: Ventilatory failure and right to left shunt in newborn infants with respiratory distress. Pediatrics 28: 17 (1961).

27. Warley, M. and Gairdner, D.: Respiratory distress syndrome of the newborn-principles in treatment. Arch. Dis. Childh. 37: 455 (1962).

28. The authors wish to thank Professor J.P.M.Tizard and Dr. R.J. Robinson for their help and criticism in preparing this paper; the Sir William Coxen Trust Fund for providing laboratory facilities; and Sister M. E. Castle and the nursing staff of the Neonatal Ward who looked after the babies.

29. Dr. Roberton is a recipient of the Percy J. Neate Research Fellowship of the Clothworkers Company. Dr. Dahlenburg was supported by a Frank J. Duval Travelling Fellowship (Melbourne).

30. Requests for reprints should be addressed to: N.R.C.Roberton, M.B., The Nuffield Neonatal Research Unit, Institute of Child Health, Hammersmith Hospital, London W. 12 (England). 